\title{
Wie ich Syndikusanwältin in der Baubranche wurde
}

\author{
Dr. Simone Reiss \\ Vorstandsmitglied des Landesverbands Saarland, Syndikusan- \\ wältin, Saarbrücken
}

Ich bin jetzt 33 Jahre alt. Als ich Abitur machte und mich für ein Studienfach entschied, hätte ich mir nie träumen lassen, 14 Jahre später als Syndikusanwältin in der Baubranche tätig sein.

Die Entscheidung für ein Jurastudium war rückblickend betrachtet fast schon zufällig. Während der Schulzeit hatte ich verschiedene Studiengänge in Betracht gezogen - Sprachen, Journalismus, Politikwissenschaften - als Grundlage für eine spätere Tätigkeit bei einer internationalen Organisation oder der Europäischen Union (EU). Kurz vor meinem Abitur las ich irgendwo, dass auch Juristen gute Chancen hätten, später in diesem Bereich eingestellt zu werden. Das Jura-Studium gilt als trocken und lernlastig - was meine eher in den MINT-Fächern aktive Verwandtschaft skeptisch anmerkte. Ich entschloss mich spontan, im Winter-Semester 2000/01 die Juristerei in Saarbrücken einfach mal „auszuprobieren“ - und war direkt begeistert. Insbesondere das Strafrecht hatte es mir - sicher auch aufgrund des Vorlesungsstils des Dozenten Prof. Radtkeangetan. Dies mündete in eine Tätigkeit als studentische Hilfskraft an seinem Lehrstuhl für Wirtschaftsstrafrecht ab dem 3. Fachsemester. Seit diesem Zeitpunkt liebäugelte ich zusätzlich mit einer Tätigkeit im Bereich des Wirtschaftsstrafrechts, bevorzugt als Staatsanwältin oder Richterin.

Nach dem 6. Fachsemester studierte ich mit einem DAADStipendium ein Jahr lang an der University of Edinburgh und belegte einen Mix aus Veranstaltungen zum schottischen (z.B. Public Law in Scotland and the UK, Evidence) und internationalen Recht (z.B. International Criminal Law), genoss aber auch intensiv das Leben in Schottland (Ceilidh und Country Dancing mit der New Scotland Dancing Society sind mir hierbei in besonders positiver Erinnerung) und einer sehr internationalen Studentengruppe. Es war eines der schönsten Jahre meines Lebens.

Zurück in Deutschland nahm ich meine Tätigkeit am Lehrstuhl für Wirtschaftsstrafrecht sowie am Europainstitut (wieder) auf. Des Weiteren standen Examensvorbereitung und Wahlfachstudium (bei mir „europäisches und internationales Recht und Menschenrechtsschutz“) auf dem Programm. Im Rahmen des Wahlfachstudiums besuchte ich u.a. die Veranstaltung „Grundzüge der Buchführung und Bilanzierung“ mein erster Berührungspunkt mit der Betriebswirtschaftslehre (BWL), die entgegen meiner Erwartung alles andere als trocken und langweilig war. Zu diesem Zeitpunkt strebte ich beruflich unverändert in Richtung EU bzw. Justizdienst (Wirtschaftsstrafrecht). Dass dieser Erstkontakt mit der BWL eine erste, fast unmerkliche Weichenstellung in Richtung meiner späteren beruflichen Tätigkeit im Bereich des Wirtschaftsrechts bzw. in der Wirtschaft sein sollte, hätte ich mir damals nie träumen lassen.
Da ich das Jahr in Edinburgh sehr genossen hatte und ein gewisses Erholungsbedürfnis nach Examensvorbereitung und Freischuss absehbar war, taktete ich die schriftlichen Prüfungen im Sommer 2005 so, dass ich eine Woche nach der letzten Wahlfachklausur in meinen zweiten Auslandsaufenthalt, diesmal an der Universidad de Zaragoza, startete. Meine Erwartungen wurden nicht enttäuscht: Die Zeit mit der Erasmus-Gruppe wurde zum unvergesslichen Highlight und verging wie im Flug. An die Examensergebnisse wurde ich erst wieder erinnert, als sie Ende November da waren. Die mündliche Prüfung fand drei Tage vor Weihnachten statt, so dass ich sie im Rahmen des Weihnachtsurlaubs miterledigen und mir mit einem guten Examen selbst das schönste Weihnachtsgeschenk machen konnte. Da ich die wenigen Veranstaltungen, die ich in Spanien besucht hatte, auch mit Schein abschließen wollte, verbrachte ich den Januar wieder in Zaragoza.

Ich hatte, weil mir auch die Arbeit am Lehrstuhl sehr gefallen hatte, alternativ zu EU und Strafjustiz eine wissenschaftliche Karriere in Erwägung gezogen und mit den Professoren Momsen (Lehrstuhl für Wirtschaftsstrafrecht, Nachfolger Prof. Radtke) und Stein (Lehrstuhl für Europa- und Völkerrecht, Direktor des Europainstituts) abgesprochen, dass ich bei ihnen zum Subventionsbetrug mit Bezügen zum Europarecht promovieren würde. Mit dem Einschub der Promotion zwischen 1. Staatsexamen und Referendariat konnte ich mich, auch wenn dieses Modell recht verbreitet ist, nicht anfreunden. Mit dem 1. Staatsexamen bzw. Jura-Diplom war ein Uni-Abschluss in Sicht. Ich hatte aber das Gefühl, dass ich mit diesem nicht wirklich etwas anfangen kann, weil die klassischen juristischen Berufsbilder das 2. Staatsexamen voraussetzen.

Daher entschied ich mich, direkt im Anschluss an meinen Erasmus-Aufenthalt das Referendariat zu beginnen und startete zum 1. Februar 2006 beim OLG Saarbrücken in die Referendarausbildung. Besonders prägend waren die Stationen in der Wirtschaftsabteilung der Staatsanwaltschaft Saarbrücken, bei Europol und bei der wirtschaftsrechtlich orientierten Kanzlei Abel und Kollegen. Die beiden ersteren Stationen zeigten mir, dass mir die Bereiche Wirtschaftsstrafrecht und Europa- bzw. internationales Recht nicht nur in der Theorie, sondern auch in der Praxis Spaß machten und bestärkten mich darin, nach Abschluss der im Anschluss an das 2. Staatsexamen geplanten Promotion beruflich einen dieser Wege zu beschreiten. Letztere Station erweiterte meinen Horizont dahingehend, dass es jenseits der von mir ins Auge gefassten Karrierewege auch im Wirtschaftsrecht spannende Betätigungsfelder gibt. Ich konnte mir zum ersten Mal in meinem Leben ernsthaft vorstellen, eventuell übergangsweise Anwältin zu werden.

Als sich mein Referendariat dem Ende zuneigte, wurde auch die Frage konkreter, wo und in welchem Umfang ich parallel zur Promotion berufstätig sein wollte. Obgleich es mir in der Wirtschaftsstrafabteilung und bei Europol sehr gut gefallen hatte, war eine wissenschaftliche Karriere nach wie vor eine 
Option. Zudem hatte ich das Wirtschaftsrecht aus anwaltlicher Sicht im Referendariat für mich entdeckt. Diese beiden Alternativen wollte ich nunmehr während eines begrenzten Zeitraums testen - und sei es nur, um Erfahrungen zu sammeln und über den Tellerrand hinauszuschauen, bevor ich den ursprünglich geplanten Weg weiterverfolge. Ich trat im Februar/März 2008 einerseits bei Prof. Momsen eine halbe Stelle als wissenschaftliche Mitarbeiterin und andererseits in der Kanzlei Abel und Kollegen eine halbe Stelle als Anwältin an. Beide Betätigungsfelder waren sehr bereichernd.

In der Kanzlei war ich zunächst vornehmlich im Insolvenzrecht tätig. Dies war nicht nur aufgrund der vielfältigen Besonderheiten der Insolvenzordnung juristisch neues Terrain für mich, das ich mit Freude erkundete. Ich erhielt auch Einblick in die betriebswirtschaftlichen Herausforderungen bei Unternehmensfortführungen in der Insolvenz bzw. sich hieran anschließende Restrukturierungsprozesse. In vielen Konstellationen wurde mir bewusst, dass die korrekte Lösung eines juristischen Problems nur ein kleiner Ausschnitt des potenziellen Betätigungsfeldes für Juristen ist und dass in der Praxis die wahre Herausforderung oft eher in der Ergründung von Sachverhalten und der geschickten taktischen Positionierung unter Berücksichtigung kaufmännischer Erwägungen liegt und dass mir dieses Tätigkeitsfeld nicht nur gefällt, sondern auch liegt.

Die Lehrstuhlarbeit - Erstellung von veranstaltungsbegleitenden Materialien, Mitarbeit an Publikationen, Abhalten von Lehrveranstaltungen - war ebenfalls vielfältig und machte, auch wegen des netten Teams, viel Spaß.

Für die Dissertation hatte ich den Bereich Verbotsirrtum und Subventionsbetrug unter Berücksichtigung europarechtlicher Einflüsse gewählt. In den ersten 16 Monaten war ich in erster Linie damit beschäftigt, mich in meinen Berufstätigkeiten zu organisieren. Irgendwo war immer viel oder Wichtiges zu tun. Ich fand zwar die Zeit, mich einzulesen, so dass die Promotion sozusagen auf Sparflamme vor sich hin köchelte, fand aber nicht den optimalen Rhythmus und war mehr damit beschäftigt, nach ein, zwei oder drei Wochen der Dissertationsuntätigkeit an den letzten Stand anzuknüpfen als tatsächlich voranzukommen. Zudem machte ich, der viele Dinge bislang eher „zugeflogen“ waren, zum ersten Mal in meinem Leben die Erfahrung, dass auch meine Kapazitäten endlich sind und beruflich mehr Aufgaben auf mich einströmen, als ich realistischerweise erledigen kann. Während dieser Zeit schrieb ich ca. 40 Seiten, von denen schlussendlich nur ca. zehn Seiten im veröffentlichen Endprodukt verblieben. Mir war klar, dass sich, wenn die Dissertation nicht versanden sollte, etwas ändern musste. Ich legte mir einen Arbeitsrhythmus zu, wonach ich morgens sehr früh anfing und in den Semesterferien immer und während des Semesters des Öfteren zunächst eine oder zwei Stunden an der Dissertation zu arbeiten und erst dann zum Tagesgeschäft überzugehen und lernte, zumindest hin und wieder nein zu sagen. Prof. Momsen gebührt großer Dank dafür, dass er immer da war, wenn ich Rücksprache brauchte, er mir aber gleichzeitig Raum gelassen hat, um mich in Dissertationsthema und Berufen zu organisieren bzw. auch inhaltlich zu finden. Knapp ein Jahr später, im Sommer 2010, war die Dissertation fertig.

Aufgrund der parallel gemachten praktischen Erfahrungen als Anwältin konnte ich mir aber nicht mehr vorstellen, ausschließlich in Forschung und Lehre tätig zu sein. Ein bisschen Lehre blieb und bleibt mir durch meine Tätigkeit als Tutorin an der Uni erhalten. Ich stockte in der Kanzlei auf eine volle Stelle auf und absolvierte Fachanwaltslehrgänge für Strafrecht sowie Bank- und Kapitalmarktrecht, wobei ich letzteren Titel seit 2012 auch führe. Zudem war ich nach wie vor im Insolvenzrecht tätig. Als ein Schwerpunkt bildete sich der Bereich (distressed) M\&A (Unternehmenskäufe (mergers and acquisitions) (aus der Insolvenz)) heraus. Zunächst war ich vor allem mit der Prüfung der rechtlichen Verhältnisse (sogenannte due diligence) befasst, teilweise auch mit der Gestaltung von Asset-Deal-Verträgen.

Im Frühjahr 2011 begann ein Mandat, das mir beruflich ganz neue Wege eröffnen sollte. Wir berieten ein expandierendes Unternehmen beim Erwerb einer in Insolvenz befindlichen Zielgesellschaft. Mir fiel die Aufgabe zu, den Insolvenzplan zu erstellen und mit den Beteiligten auszuhandeln. Weitere Mandate aus dem M\&A-Segment und anderen Bereichen folgten. Mandant war die Baugruppe Peter Gross. Seit dem 1. Januar 2014 bin ich bei der Baugruppe Peter Gross als Syndikusanwältin tätig. Ich betreue fünf Niederlassungen in erster Linie im Baurecht, also vor allem bei der Vertragsgestaltung und bei Streitfällen. In meinen Zuständigkeitsbereich fallen zudem Compliance, AGB, Muster, Insolvenzen Dritter, Unternehmenskäufe, sowie sonstige Rechtsfragen. So sehr mir die vorherige Tätigkeit in einer Kanzlei gefallen hat, habe ich vor allem bei Unternehmensinsolvenzen immer ein wenig bedauert, sozusagen zur Besatzung des „Notarztwagens“ zu gehören. Wenn der „Patient“ noch zu retten ist, gibt man ihn nach einer ersten Stabilisierung des Geschäftsbetriebs in hoffentlich glücklichere Hände ab. Von meiner neuen Tätigkeit erhoffe ich mir vor allem, Dinge mit Blick auf einen längerfristigen Zeithorizont zu gestalten.

Ich für mich kann nur sagen, dass man Wege zu einem spannenden und beglückenden Beruf nicht auf dem Reißbrett entwerfen muss. Sicher sollte man sich Ziele setzen und diese verfolgen. Parallel sollte man sich aber auch eine Offenheit für Alternativen bewahren und spontan Gelegenheiten ergreifen. Blicke über den Tellerrand, sei es im Rahmen von Auslandsaufenthalten, sei es in Bezug auf vorher nicht in Betracht gezogene Tätigkeitsfelder, schaden nie. Ich habe oft von Dingen, die ich zufällig oder aus Interesse am Wegesrand mitnahm, einige Biegungen später in ganz anderem Kontext profitiert. Wohin mich ein beruflicher und privater Weg noch führen wird, weiß ich nicht. Langweilig wird es sicher nicht. Und am Rande - gegen eine der vielbeschworenen gläsernen Decken bin ich bislang nicht gestoßen. Daher - nur Mut! 